\title{
Diagnoses of Cardiovascular Disease or Substance Addiction/ Abuse in US Adults Treated for ADHD with Stimulants or Atomoxetine: Is Use Consistent with Product Labeling?
}

\author{
Kathleen A. Fairman ${ }^{1}$ - Lindsay E. Davis ${ }^{1} \cdot$ Alyssa M. Peckham ${ }^{1} \cdot$ David A. Sclar $^{1}$
}

Published online: 5 January 2018

(C) The Author(s) 2018. This article is an open access publication

\begin{abstract}
Background Among US adults, utilization of pharmacotherapy for attention-deficit hyperactivity disorder (ADHD) has increased more than ninefold since 1995-1996. Potential contraindications to ADHD pharmacotherapy include serious cardiovascular disease (CVD) and, for stimulants, addictions and bipolar disorder (BPD). Objective To assess the prevalence of potential contraindications among adults treated with ADHD pharmacotherapy.

Methods A retrospective cohort analysis was performed using the Truven Health MarketScan ${ }^{\circledR}$ database. Subjects filled $\geq 1$ prescription for atomoxetine or $\geq 1$ stimulant in 2014-2015, were aged 18-64 years, commercially insured throughout observation, and diagnosed with ADHD on two or more medical claims. Diagnoses and medical procedures were measured in the 12 months prior to pharmacotherapy initiation. Metrics included serious CVD (cardiomegaly, cardiomyopathy, cerebrovascular occlusion, congestive heart failure, myocardial infarction, pacemaker, or valvular
\end{abstract}

disorder) and any CVD (serious CVD, other atherosclerotic CVD, arrhythmia, congenital heart anomaly, or hypertensive heart disease). Rates of substance addiction or abuse were measured in a range to address nonspecific diagnostic coding.

Results Only $2.0 \%$ of treated adults $(n=91,588)$ had one or more diagnosis indicating serious CVD. CVD prevalence increased monotonically with age. Of patients aged 55-64 years $(n=5,237), 7.2 \%$ had serious CVD; $15.9 \%$ had any CVD; and $1.9 \%$ had been hospitalized with one or more CVD. Of patients treated with stimulants ( $n=87,167), 11.3-18.5 \%$ were diagnosed with addiction/ abuse and $4.1 \%$ with BPD.

Conclusions CVD prevalence is generally low among adults using ADHD medication but increases with age. Although difficult to estimate precisely, the rate of addiction/abuse among stimulant-treated patients appears unexpectedly high. Further research should assess cardiovascular events and other potential harms associated with contraindicated use in high-risk adults.
Electronic supplementary material The online version of this article (https://doi.org/10.1007/s40801-017-0129-2) contains supplementary material, which is available to authorized users.

Kathleen A. Fairman

kfairm@midwestern.edu

1 Department of Pharmacy Practice, College of Pharmacy, Midwestern University-Glendale, 19555 N. 59th Avenue, Glendale, AZ 85308, USA 


\section{Key Points}

This study of potential contraindications to pharmacotherapy for attention-deficit hyperactivity disorder (ADHD) in commercially insured US adults found that, measured in the 12 months prior to initiation of treatment with stimulants or atomoxetine in 2014-2015, prevalence rates of serious cardiovascular disease were $7.2 \%$ among those aged 55-64 years and 3.6\% among those aged $45-54$ years.

Of adults initiating treatment with stimulants, $11.3-18.5 \%$ had been diagnosed in the past year with substance addiction or potential abuse.

In light of rapid growth in diagnosis and pharmacologic treatment for ADHD in recent years, a study of adverse drug events among adults at highest risk - those who are older and/or have a potential contraindication to pharmacotherapy-is needed.

\section{Introduction}

In the past two decades, prevalence rates of diagnosis and pharmacologic treatment for attention-deficit hyperactivity disorder (ADHD) have increased exponentially among United States (US) adults [1-6]. One study of National Ambulatory Medical Care Survey (NAMCS) data found that per 1,000 office-based physician visits made by US adults aged $\geq 20$ years, rates of ADHD diagnosis and pharmacologic treatment increased by 52.6 and $35.2 \%$, respectively, from 2008-2009 to 2012-2013 [6]. Moreover, from 1995-1996 to 2007-2008, the population-adjusted rate of physician visits at which ADHD pharmacotherapy was prescribed to adults increased fivefold; and by 2012-2013, a more than ninefold increase had occurred, from 1.9 to 11.4 visits per 1000 US adults $[2,5,6]$.

It has been suggested that these changes are attributable to expansion of the diagnostic criteria for ADHD in the Diagnostic and Statistical Manual of Mental Disorders (DSM)-5 in 2013 [7], as well as the launch of new medications and formulations (e.g., chewables, sustained-release tablets, patches, and new molecular entities) in recent years $[5,8,9]$. Along with these expansions, two factors complicate the management of pharmacotherapy for the rapidly growing population of US adults diagnosed with ADHD.
First, ADHD medications increase diastolic blood pressure (BP), systolic $\mathrm{BP}$, and heart rate by amounts that are generally considered modest but potentially clinically significant in patients with pre-existing cardiovascular disease (CVD) or CVD risk factors [10]. For this reason, stimulants, which are the most common and guidelinerecommended medications to treat ADHD [6, 11, 12], carry US Food and Drug Administration (FDA) labeled warnings for serious cardiovascular events, including sudden death, stroke, and myocardial infarction (MI) [13]. Specifically, product labels state that stimulants "generally should not be used" in patients with "serious structural cardiac abnormalities, cardiomyopathy, serious heart rhythm abnormalities, or other serious cardiac problems that may place them at increased vulnerability to the sympathomimetic effects of a stimulant drug"; and that "caution is indicated" in treating patients with pre-existing hypertension [14-17]. Atomoxetine, a non-stimulant therapeutic option in the treatment of ADHD, carries a similar warning [18]. Because older age increases the risk of CVD [19], increased pharmacovigilance for cardiovascular risk factors and events is appropriate as the population of adults treated with ADHD pharmacotherapy expands and ages [20].

Second, prescription stimulant medications are the subject of a growing degree of public health concern about addiction and related adverse medical events [21, 22]. From 2005 to 2011, the number of US emergency department (ED) visits attributable to non-medical use of stimulant pharmaceuticals by young adults (aged 18-34 years) more than tripled, from 5,605 to 22,949 [23]. Additionally, for all age groups combined, more than 31,000 ED visits were made because of ADHD medications in 2010 in the US [24]. Because these increases have taken place primarily among adults, rather than among children and adolescents, the US Drug Abuse Warning Network (DAWN) has identified "a need for increased attention toward ... diversion and misuse among adults ... as treatment for ADHD among adults becomes more widespread" [24]. FDA product labels for stimulants indicate that they should "be given cautiously to patients with a history of drug dependence or alcoholism" [14-17].

In theory, the publication of evidence and of evidencebased guidelines, such as those currently available for adult ADHD, should be reflected in the pharmacotherapies prescribed for the disorder in real-world practice. However, prescribing behaviors do not consistently respond to the available base of evidence. For example, there was no discernible decline in prescribing of pharmacotherapy for pediatric ADHD after the FDA issued a warning in 2006 about cardiac and psychiatric risks of stimulants [25]. Additionally, previous work has documented potentially contraindicated prescribing of a variety of medication classes, for example, antidepressants, statins, and serotonin 
receptor agonists [26-29]. Thus, it is important to examine the rate of potentially contraindicated prescribing systematically, particularly when the patient population treated with a given class of medication changes over time, as it has in ADHD.

Current evidence about potential contraindications in the prescribing of ADHD medications to adults is limited. One analysis of adults with a new diagnosis of ADHD in 2006 or 2007 examined the likelihood of ADHD pharmacotherapy use as a function of baseline cardiovascular risk [30]. Another assessed the risk of serious cardiovascular events, controlling for baseline cardiovascular risk, in patients treated from 1986 to 2005 [31]. The time periods studied in both these analyses preceded promulgation of the DSM-5 [7, 30, 31]. Additional studies of cardiovascular events associated with pharmacotherapy for adult ADHD have either excluded patients with high-risk conditions from the sample or used a propensity-matched cohort design, thereby making it difficult to determine the percentage of stimulant- or atomoxetine-treated patients who are potentially at increased risk of adverse events [32, 33].

The present study addressed this gap in available information by profiling the relevant clinical histories of cohorts of adult patients filling prescriptions for either stimulants or atomoxetine to treat ADHD in 2014-2015. Specifically, the study examined rates of (1) CVD and CVD risk factors, which are potential contraindications for both stimulants and atomoxetine; and (2) substance addiction or abuse, bipolar disorder, and glaucoma, which are potential contraindications for stimulants [14-18].

\section{Methods}

\subsection{Study Design and Data}

The study was a retrospective cohort analysis of patients identified using the Truven MarketScan ${ }^{\circledR}$ Commercial Claims and Encounters database, which includes claims for all healthcare services (medical care and prescription medications) delivered to approximately 50 million commercially insured enrollees each year. The MarketScan database, which has been used in more than 1400 published studies of US healthcare, is fully compliant with Health Insurance Portability and Accountability Act (HIPAA) standards [34]. Data are obtained by Truven Health from employers and health insurance plans, cleaned for quality and accuracy, and de-identified using encrypted case numbers for research purposes. The database includes the Truven Health Red Book ${ }^{\circledR}$ table, available to licensed users, which matches national drug code numbers to medication product information, including generic name. The study was deemed exempt from Institutional Review
Board (IRB) review by the Midwestern University IRB committee.

\subsection{Study Sample}

The sample was drawn from claims for all filled prescriptions and medical services with dates of service from 1 January 2013, through 31 December 2015. All patients aged 18-64 years, as measured on the first enrollment date in each calendar year, who met the criteria listed below were included in the sample (Fig. 1):

- Filled $\geq 1$ prescription for an ADHD medication (amphetamines, atomoxetine, dexmethylphenidate or methylphenidate), identified using generic product name, in either 2014 or 2015. Use of lisdexamfetamine did not qualify patients for the sample because it has a labeled indication for binge-eating disorder [35], an obesity-related condition that is associated with CVD risk factors including diabetes, hypertension, and dyslipidemias [36, 37].

- Were continuously enrolled for healthcare benefits for $\geq 12$ months prior to the first ADHD medication claim date (index date), creating a sample of new users (i.e., after $\mathrm{a} \geq 12$-month "washout" period). To allow for $\geq 12$ months of eligibility prior to the index medication claim, the earliest index date was 1 January 2014.

- Had two or more medical claims with a diagnosis of ADHD (International Classification of Diseases [ICD]9 code of 314.xx or ICD-10 code of F90.xx) at any time. This sampling step excluded patients who used the medications either off-label or for other FDAlabeled uses, such as narcolepsy or obesity [38]. The application of a "two or more" claim rule for ADHD diagnosis was used to exclude patients whose claims reflect "rule-out" diagnoses or coding errors, a technique that is common in claims database analyses [39-41].

- One or more claim with an ADHD diagnosis either preceded the index date or followed it by no more than 90 days. The 90-day standard was used to link medication therapy to diagnosis, while allowing for minor variations in practice patterns (e.g., empirical treatment followed by diagnosis) or billing practices.

\subsection{Measurement of Potential Contraindications and Co-morbidities}

Medical diagnoses and procedures were measured and calculated as prevalence rates (i.e., percentages: total number diagnosed divided by total number of patients) during the 12-month time period preceding the index date. 
Fig. 1 Sample selection flowchart. Amphetamines (amphetamine salt combination, amphetamine sulfate, dextroamphetamine sulfate, hydroxyamphetamine, methamphetamine), dexmethylphenidate, or methylphenidate. $A D H D$ attention-deficit hyperactivity disorder
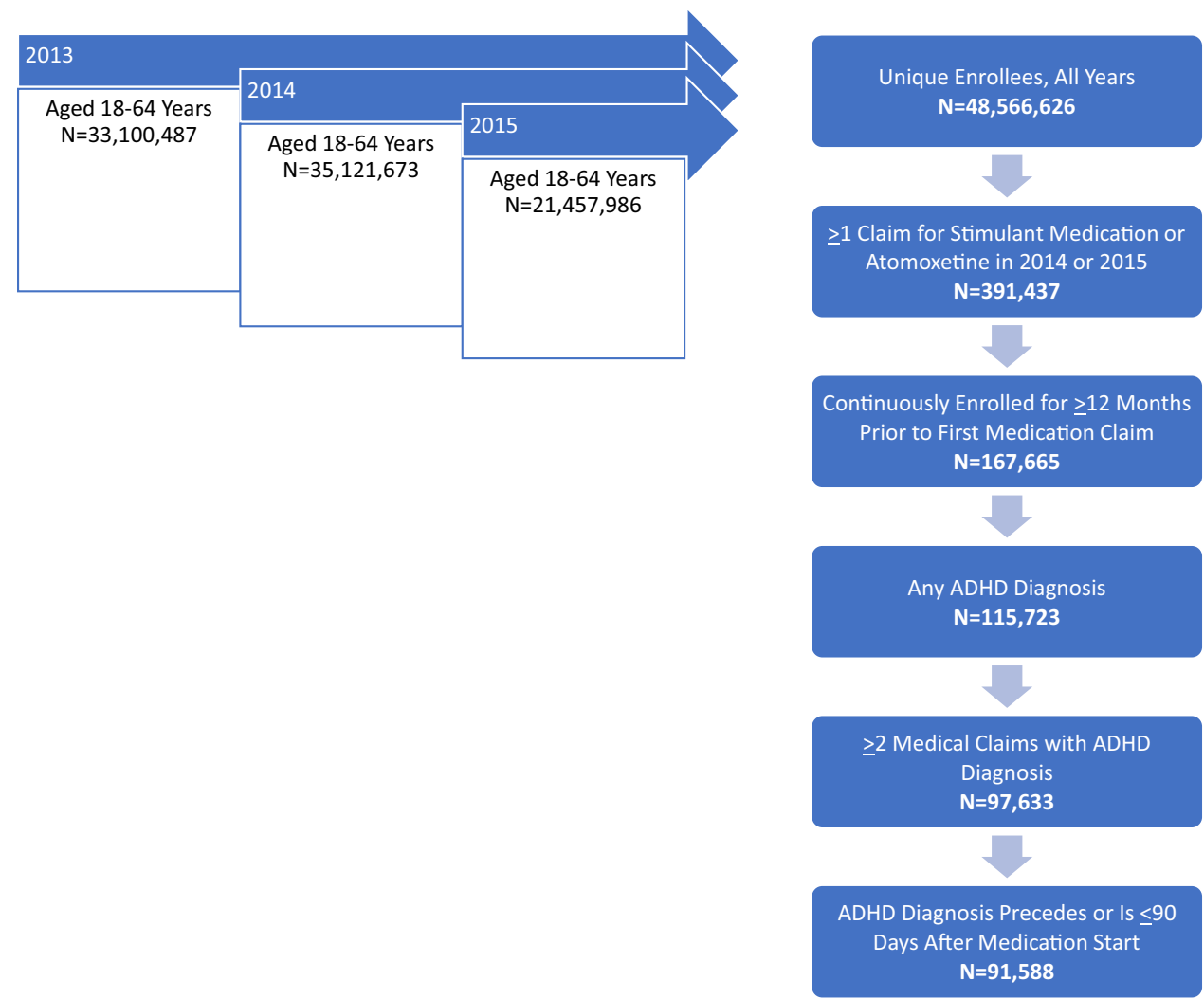

Diagnoses were measured in any of the first four diagnosis fields reported on ambulatory claims (outpatient hospital department, ED, and physician office), and in these four fields plus the primary diagnosis field and diagnosis-related group (DRG) codes on inpatient hospital claims. Additionally, detoxification services (identified by revenue codes, place of service codes, and Health Care Common Procedural Coding System codes) were used to identify addiction/abuse; and revascularization (i.e., percutaneous transluminal angioplasty, stenting, balloon angioplasty, and coronary artery bypass grafting) was measured using procedure codes for all treatment settings and DRG codes for inpatient admissions.

Potential contraindications were measured in three categories: CVD, addiction/abuse, and other (bipolar disorder and glaucoma). Within the CVD category, a measure of serious CVD_intended to represent the FDA's warning language for "serious structural cardiac abnormalities, cardiomyopathy, serious heart rhythm abnormalities, CAD or other serious cardiac problems"-was defined as cardiomegaly, cardiomyopathy, cerebrovascular occlusion, congestive heart failure, myocardial infarction, pacemaker, or valvular disorder [14-18]. CVD was defined as serious CVD, other atherosclerotic CVD, arrhythmia, congenital heart anomaly, or hypertensive heart disease. Although not included in the summary measure of CVD, prevalence rates of diabetes, hyperlipidemia, and hypertension were reported because these are risk factors for CVD events [42]. Diagnosis codes are shown in Online Appendix A, and procedure codes are shown in Online Appendix B.

Sensitivity analyses were performed to address one area of ambiguity in diagnostic coding on medical claims. Specifically, the a priori definition of abuse/addiction included diagnoses of V58.69 (ICD-9, "long-term (current) use of other medications") and Z79.891 (ICD-10, "longterm (current) use of opiate analgesic") [43, 44]. This approach was used for several reasons: (1) Previous research has documented variations in diagnostic coding specificity in administrative data, particularly for conditions that are stigmatized and/or difficult to diagnose [45-47]; (2) The ICD-9 code description for long-term medication use specifically refers to methadone, opiate analgesics, and "other high-risk medications" [48]; and, (3) Of all inpatient claims for the study sample that included a nonspecific code, $97 \%$ also were coded for a specific diagnosis of abuse or addiction.

Nonetheless, because the ICD-9 code for long-term medication use was indeterminate as to specific drug, a set of post hoc sensitivity analyses limited the addiction/abuse prevalence indicator to patients with either (1) a specific diagnosis of abuse/addiction during the 12-month pretreatment time frame or (2) a code for long-term medication/opiate use and a procedure code indicating a laboratory test for a specific controlled substance at any time 
prior to treatment initiation. This choice was made because a post hoc exploratory analysis showed that $36 \%$ of outpatient claims with the nonspecific code lacked a substance abuse diagnosis; and, of those, $25 \%$ were laboratory claims for specific controlled substance tests. Codes for drug screens that were not specific as to substance were not included in this measure. The codes and substances captured in this assessment are shown in online Appendix C.

To assess the relationship between potential contraindications and aging, the percentages of patients with each diagnosis were calculated not only overall, but also by age group. To determine whether risk from potential contraindications increases ordinally (i.e., monotonically) with age, between-group differences were tested using the Mantel-Haenszel (linear-by-linear association) test for trend [49]. To produce nationally representative estimates, all results were weighted for the sample-to-population ratio across strata formed on sex, age group, region, and policyholder status (i.e., enrollee vs. dependent), using a method and strata population sizes provided by Truven Health. The total sample size after weighting was held to the original (pre-weighting) cohort size by applying a constant to all strata weights. All calculations were performed using SPSS v24.0 (IBM Corporation, Armonk, NY, USA) at an a priori alpha (critical $P$ value) of 0.05 .

\section{Results}

Among all adult patients in the sample who were treated for ADHD with stimulants or atomoxetine in 2014-2015 $(n=91,588)$, most individual CVD diagnoses were rare ( $\leq 0.5 \%$ prevalence), and almost no patients had a history of pacemaker implantation or revascularization (Table 1). Within the sample overall, $2.0 \%$ had a diagnostic history potentially indicating serious CVD (cardiomegaly, cardiomyopathy, cerebrovascular occlusion, congestive heart failure, myocardial infarction, pacemaker, or valvular disorder); 1.6\% had ASCVD (angina, cerebral occlusion, MI, peripheral arterial disease, transient ischemic attack (TIA), revascularization, or other ASCVD); and 5.5\% had some form of CVD (serious CVD, other atherosclerotic CVD, arrhythmia, congenital heart anomaly, or hypertensive heart disease). Only $0.6 \%$ of patients treated with any ADHD medication, and $1.3 \%$ of patients treated with atomoxetine, were hospitalized with a diagnosis of any CVD condition in the 12 months prior to the start of pharmacotherapy.

Like the individual CVD diagnoses, co-morbidities were relatively uncommon in the sample overall (Table 1). For example, hyperlipidemia and hypertension were each diagnosed in $11.5 \%$ of patients, and diabetes in $3.1 \%$.
Chronic kidney disease of at least moderate severity (Stages 3 or higher) was rare $(0.1 \%$ of the sample).

A history of addiction/abuse as defined in the a priori analysis plan was much more common $-18.8 \%$ of the sample overall, $18.5 \%$ of patients treated with stimulants ( $\mathrm{n}=87,167)$, and $23.9 \%$ of patients treated with atomoxetine $(n=7051$; Table 1$)$. However, measurement of addiction/abuse prevalence was sensitive to the inclusion of nonspecific codes for long-term medication use. In the post hoc sensitivity analysis with the modified (stricter) definition of addiction/abuse, prevalence rates were $11.7 \%$ for the sample overall, $11.3 \%$ for stimulant-treated patients, and $17.1 \%$ for patients treated with atomoxetine. Of patients treated with stimulants, $4.1 \%$ were diagnosed with bipolar disorder and $0.6 \%$ with glaucoma.

Despite the generally low rate of potential contraindications and co-morbidities in the sample overall, the prevalence of all CVD diagnoses and risk factors increased monotonically with age, as expected (Table 2). Of patients aged $45-54$ years $(n=12,801), 8.7 \%$ had any CVD; $3.6 \%$ had serious CVD; and $1.0 \%$ were hospitalized with a diagnosis of CVD in the 12 months prior to the start of ADHD pharmacotherapy. These rates were nearly doubled in patients aged 55-64 years $(n=5237): 15.9 \%$ had any CVD; $7.2 \%$ had serious CVD; and $1.9 \%$ were hospitalized with a diagnosis of CVD in the 12 months prior to the start of ADHD pharmacotherapy (all $P<0.001$ ).

Rates of glaucoma and hypertension were also considerably elevated in older patients (Table 2). Specifically, among those aged 55-64 years compared with patients in the youngest age group (aged 18-24 years, $n=30,499$ ) the rate of glaucoma was multiplied 18 -fold (3.6 vs. $0.2 \%$, respectively); and the rate of hypertension was multiplied $>20$-fold ( 40.1 vs. $2.0 \%$, respectively). Rates of diabetes and hyperlipidemia were similarly elevated: diabetes $>14$-fold and hyperlipidemia $>20$-fold (all $P<0.001)$.

\section{Discussion}

In an analysis of commercially insured adults treated with pharmacotherapy for ADHD in 2014-2015, we found prevalence rates of CVD and CVD risk factors that were generally low overall but markedly elevated with advancing age, particularly among patients aged 55-64 years. In that age group, $15.9 \%$ were diagnosed with CVD and $7.2 \%$ with serious CVD; and 40\% had diagnosed hypertension. Additionally, we found a high rate of pre-existing addiction/abuse in the sample overall. Among adults treated with stimulants, $11.3-18.5 \%$ were diagnosed with some form of addiction or potential abuse in the 12 months prior to the start of ADHD pharmacotherapy. To the knowledge of these authors, these 
Table 1 Demographic characteristics and 12-month pre-treatment diagnoses, patients aged 18-64 years treated for ADHD with stimulants or atomoxetine in 2014-2015

\begin{tabular}{|c|c|c|c|}
\hline & Stimulants & Atomoxetine & Whole sample $^{\mathrm{a}}$ \\
\hline$N$ & 87,167 & 7051 & 91,588 \\
\hline \multicolumn{4}{|l|}{ Age (years) } \\
\hline Mean (median) & $33(31)$ & $34(32)$ & $33(31)$ \\
\hline \multicolumn{4}{|l|}{ Age group (\%) } \\
\hline $18-24$ & 33.3 & 33.0 & 33.3 \\
\hline $25-34$ & 26.0 & 22.5 & 25.8 \\
\hline $35-44$ & 21.2 & 21.6 & 21.2 \\
\hline $45-54$ & 13.9 & 16.3 & 14.0 \\
\hline $55-64$ & 5.6 & 6.6 & 5.7 \\
\hline Female $(\%)$ & 51.3 & 48.0 & 51.1 \\
\hline \multicolumn{4}{|l|}{ Cardiovascular disease $(\%)$} \\
\hline Angina $^{\mathrm{b}}$ & 0.2 & 0.3 & 0.2 \\
\hline Arrhythmia/tachycardia & 3.0 & 5.0 & 3.1 \\
\hline Cardiomegaly $^{\mathrm{c}}$ & 0.3 & 0.4 & 0.3 \\
\hline Cardiomyopathy $^{\mathrm{c}}$ & 0.1 & 0.3 & 0.2 \\
\hline Cerebral occlusion ${ }^{\mathrm{b}, \mathrm{c}}$ & 0.5 & 0.6 & 0.6 \\
\hline Congenital heart anomaly & 0.3 & 0.4 & 0.3 \\
\hline Congestive heart failure $^{c}$ & 0.1 & 0.3 & 0.1 \\
\hline Hypertensive heart disease & 0.3 & 0.4 & 0.3 \\
\hline Myocardial infarction ${ }^{\mathrm{b}, \mathrm{c}}$ & 0.1 & 0.1 & 0.1 \\
\hline Peripheral arterial disease ${ }^{\mathrm{b}}$ & 0.2 & 0.2 & 0.2 \\
\hline Transient ischemic attack ${ }^{\mathrm{b}}$ & 0.2 & 0.3 & 0.2 \\
\hline Valvular disorder $^{\mathrm{c}}$ & 1.0 & 1.4 & 1.0 \\
\hline \multicolumn{4}{|l|}{ Cardiac procedures $(\%)$} \\
\hline Pacemaker (facility claims) ${ }^{\mathrm{c}}$ & 0.0 & 0.1 & 0.0 \\
\hline Revascularization (facility claims) ${ }^{\mathbf{b}}$ & 0.0 & 0.1 & 0.0 \\
\hline \multicolumn{4}{|l|}{ Cardiovascular summary measures (\%) } \\
\hline Any CVD & 5.4 & 8.2 & 5.5 \\
\hline Inpatient stay associated with CVD & 0.6 & 1.3 & 0.6 \\
\hline Serious CVD & 1.9 & 2.8 & 2.0 \\
\hline Any ASCVD $^{\mathrm{b}}$ & 1.6 & 2.1 & 1.6 \\
\hline \multicolumn{4}{|l|}{ Other potential contraindications (\%) } \\
\hline Addiction/abuse or long-term medication/opiate use & 18.5 & 23.9 & 18.8 \\
\hline Addiction/abuse or long-term medication/opiate use with testing for abusable substances & 11.3 & 17.1 & 11.7 \\
\hline Bipolar disorder & 4.1 & 7.8 & 4.3 \\
\hline Glaucoma & 0.6 & 0.6 & 0.6 \\
\hline \multicolumn{4}{|l|}{ Co-morbidities $(\%)$} \\
\hline Diabetes & 3.1 & 3.9 & 3.1 \\
\hline Moderate-to-severe CKD & 0.1 & 0.2 & 0.1 \\
\hline Hyperlipidemia & 11.3 & 14.8 & 11.5 \\
\hline Hypertension & 11.3 & 14.5 & 11.5 \\
\hline Seizure disorder & 0.7 & 1.2 & 0.7 \\
\hline
\end{tabular}

$\overline{A D H D}$ attention-deficit hyperactivity disorder, $A S C V D$ atherosclerotic cardiovascular disease, $C K D$ chronic kidney disease, $C V D$ cardiovascular disease

${ }^{a}$ Because subcohorts are not mutually exclusive, sum of the subcohort counts exceeds total sample size

${ }^{b}$ ASCVD. In addition to the specific diagnoses shown, the ASCVD summary measure includes diagnosis codes for atherosclerosis or ischemic heart disease

${ }^{\mathrm{c}}$ Serious CVD 
Table 2 Demographic characteristics and 12-month pre-treatment diagnoses, patients aged 18-64 years treated for ADHD with stimulants or atomoxetine, by age group

\begin{tabular}{|c|c|c|c|c|c|}
\hline & $18-24$ years & 25-34 years & $35-44$ years & $45-54$ years & $55-64$ years \\
\hline$N^{\mathrm{a}}$ & 30,499 & 23,633 & 19,419 & 12,801 & 5,237 \\
\hline Female $(\%)^{*}$ & 45.1 & 47.8 & 56.4 & 60.5 & 58.7 \\
\hline \multicolumn{6}{|l|}{ Cardiovascular disease } \\
\hline Angina $^{\mathrm{b}, *}$ & 0.0 & 0.0 & 0.1 & 0.5 & 0.8 \\
\hline Arrhythmia/tachycardia* & 2.4 & 2.8 & 3.2 & 3.7 & 6.0 \\
\hline Cardiomegaly ${ }^{\mathrm{c}, *}$ & 0.1 & 0.1 & 0.3 & 0.6 & 1.0 \\
\hline Cardiomyopathy ${ }^{\mathrm{c}, *}$ & 0.1 & 0.1 & 0.2 & 0.2 & 0.6 \\
\hline Cerebral occlusion ${ }^{\mathrm{b}, \mathrm{c}, *}$ & 0.2 & 0.3 & 0.6 & 1.1 & 2.6 \\
\hline Congenital heart anomaly & 0.3 & 0.3 & 0.2 & 0.3 & 0.3 \\
\hline Congestive heart failure ${ }^{\mathrm{c}, *}$ & 0.0 & 0.1 & 0.2 & 0.3 & 0.7 \\
\hline Hypertensive heart disease* & 0.0 & 0.1 & 0.4 & 0.5 & 1.4 \\
\hline Myocardial infarction ${ }^{\mathrm{b}, \mathrm{c}, *}$ & 0.0 & 0.0 & 0.1 & 0.3 & 0.6 \\
\hline Peripheral arterial disease ${ }^{\mathrm{b}, *}$ & 0.0 & 0.1 & 0.1 & 0.5 & 1.0 \\
\hline Transient ischemic attack $^{\mathrm{b}, *}$ & 0.0 & 0.1 & 0.2 & 0.5 & 0.6 \\
\hline Valvular disorder ${ }^{\mathrm{c}, *}$ & 0.5 & 0.6 & 1.2 & 1.6 & 3.3 \\
\hline \multicolumn{6}{|l|}{ Cardiovascular summary measures* } \\
\hline Any CVD & 3.3 & 4.2 & 5.9 & 8.7 & 15.9 \\
\hline Inpatient stay associated with CVD & 0.4 & 0.6 & 0.6 & 1.0 & 1.9 \\
\hline Serious CVD & 0.8 & 1.1 & 2.3 & 3.6 & 7.2 \\
\hline Any ASCVD & 0.4 & 0.6 & 1.4 & 3.7 & 8.3 \\
\hline \multicolumn{6}{|l|}{ Cardiac procedures* } \\
\hline Pacemaker $^{\mathrm{c}}$ & 0.0 & 0.0 & 0.1 & 0.1 & 0.1 \\
\hline Revascularization $^{\mathrm{b}}$ & 0.0 & 0.0 & 0.1 & 0.1 & 0.2 \\
\hline \multicolumn{6}{|l|}{ Potential contraindications* } \\
\hline Addiction/abuse or long-term medication/opiate use & 16.9 & 18.1 & 20.0 & 21.3 & 23.2 \\
\hline $\begin{array}{l}\text { Addiction/abuse or long-term medication/opiate use with testing for } \\
\text { abusable substances }\end{array}$ & 11.0 & 11.9 & 12.3 & 11.7 & 11.7 \\
\hline Bipolar disorder & 3.6 & 4.1 & 4.5 & 5.2 & 5.7 \\
\hline Glaucoma & 0.2 & 0.3 & 0.5 & 1.2 & 3.6 \\
\hline \multicolumn{6}{|l|}{ Co-morbidities } \\
\hline Diabetes* & 0.9 & 1.5 & 3.7 & 7.0 & 12.2 \\
\hline Moderate-to-severe CKD* & 0.0 & 0.0 & 0.1 & 0.3 & 0.9 \\
\hline Hyperlipidemia* & 2.0 & 6.1 & 15.2 & 26.7 & 40.9 \\
\hline Hypertension* & 2.0 & 6.7 & 15.2 & 25.6 & 40.1 \\
\hline Seizure disorder & 0.7 & 0.8 & 0.6 & 0.6 & 0.8 \\
\hline
\end{tabular}

$\overline{A D H D}$ attention-deficit hyperactivity disorder, $A S C V D$ atherosclerotic cardiovascular disease, $C K D$ chronic kidney disease, $C V D$ cardiovascular disease

${ }^{*} P<0.001$, linear-by-linear association test

${ }^{\text {a }}$ Sum of cell counts exceeds sample size by 1 because of the application of sample weights

${ }^{\mathrm{b}}$ ASCVD. In addition to the specific diagnoses shown, the ASCVD summary measure includes diagnosis codes for atherosclerosis or ischemic heart disease

${ }^{\mathrm{c}}$ Serious CVD

findings represent the first "real-world" assessment of potentially contraindicated prescribing of atomoxetine and stimulants for adults since expansion of the diagnostic criteria for ADHD in the DSM-5.
Prevalence rates for CVD and CVD risk factors observed in the present study are generally similar to those observed in research conducted in earlier time periods, despite some methodological differences. In a retrospective 
analysis of health records for commercially insured and Medicaid-enrolled adults (aged 25-64 years, time period 1986-2007), Habel et al. found prevalence rates of $14.8 \%$ for hypertension, $18.7 \%$ for hyperlipidemia, $1.2 \%$ for stroke/TIA, and $0.2 \%$ for MI, measured in the 12 months prior to the start of ADHD pharmacotherapy [31]. In the subset of patients aged 25-64 in the present analysis, we found prevalence rates of $16.2 \%$ for hypertension, $16.3 \%$ for hyperlipidemia, $0.8 \%$ for cerebral occlusion, $0.3 \%$ for TIA, and $0.1 \%$ for MI.

Similarly, Gerhard et al. used a claims database to study adults (aged 21-64 years) with a new diagnosis of ADHD in 2006-2007, followed through March 2008 [30]. In that study's subcohort of patients treated with stimulants or atomoxetine, $8.8 \%$ had either any cardiovascular condition or diagnosed hypertension in the 12 months prior to initial diagnosis, with prevalence rates increasing monotonically from $2.7 \%$ among those aged 21-29 years to $22.0 \%$ among those aged 46-64 years (percentages calculated from counts shown in study report).

A notable difference between our results and those of Habel et al. is the markedly higher rate of diagnosed substance addiction or abuse observed in the present study sample: $11.7-18.8 \%$ compared with $5.2 \%$ observed by Habel et al. for "alcohol/substance abuse" in 1986-2007 [31]. It is possible that methodological differences, such as the use of health records by Habel et al. and claims data in the present study, contribute to the observed increase in the addiction/abuse prevalence rate. However, given the marked increase in use and abuse of controlled prescription medications that has been noted by US public health organizations in recent years [21, 23, 24], it appears more likely that the results of the present study are a manifestation of the public health crisis associated with increases in the prevalence of prescription medication abuse in the US [50]. In one study of U.S. college students who were asked to self-report use of stimulants in the previous year, $5.4 \%$ in 2003 and $9.3 \%$ in 2013 reported non-medical use, whereas $1.9 \%$ in 2003 and $4.7 \%$ in 2013 reported medical use [51]. It is also possible that patients with pre-existing addictions were erroneously diagnosed with ADHD, because substance abuse disorders complicate the process of differential diagnosis in patients presenting with symptoms of $\mathrm{ADHD}$, such as restlessness, inattention, or impulsivity [52].

Present study findings suggest that prescribers were aware that a history of addiction places adults at risk when treated with stimulants [53], as addiction was more prevalent among atomoxetine-treated patients (17.1-23.9\%) than among those treated with stimulants (11.3-18.5\%). However, it is somewhat puzzling that CVD was also more prevalent among atomoxetine- than stimulant-treated patients ( 8.2 vs. $5.4 \%$, respectively), because product labels for both atomoxetine and stimulants have similar warnings for cardiovascular events.

In considering the policy implications of these findings, it is appropriate to take into account the concerns underlying the FDA's Drug Safety and Risk Management Advisory Committee 2006 recommendation that a "blackbox" warning for cardiovascular effects be added to stimulant product labels, although the FDA did not adopt the recommendation. As described by a committee advisor in an editorial published later that year, one factor considered by the committee was the "rapid increase in exposure" associated with expanding prevalence of ADHD diagnosis and stimulant use in adults [54]. Because this expansion would result in "the administration of potent sympathomimetic agents to millions of Americans," the editorialist noted, the committee "sought to emphasize more selective and restricted use, while increasing awareness of potential hazards" [54]. In the decade that has passed since that editorial was written, exposure to ADHD pharmacotherapy has rapidly increased among US adults, making the Committee's concerns even more cogent today.

However, previous studies of the cardiovascular safety of pharmacotherapy for adult ADHD either controlled for baseline CVD or excluded patients with CVD from the sample [31-33]. Although these methodological features are standard techniques used to control for pre-existing disease in a population-wide assessment of adverse drug effects, the present study results may suggest the need for a more targeted approach: assess hazards in those subpopulations most at risk. Specifically, among adult patients treated for ADHD with stimulants or atomoxetine, future research should assess the prevalence of (1) cardiovascular events in those who are aged 55 years or older or have a pre-existing CVD, perhaps with special emphasis on those with both risk factors; and (2) sequelae of addiction/abuse (e.g., hospitalization for adverse drug reactions or mortality) in those with pre-existing histories of substance use disorder.

\subsection{Limitations}

Several limitations of the present study should be noted. First, the study did not assess the effects of long-term medication exposure occurring prior to the start of the study, for example, use throughout childhood in a patient who is now an adult. Similarly, diagnoses of contraindications could have been made either prior to the 12-month measurement time frame used in the present study, or for services paid out-of-pocket by patients and therefore not recorded in insurance claims. Thus, the calculated prevalence rates may be underestimated.

Second, the study was limited to commercially insured enrollees aged 18-64 years; its results may not be 
applicable to those enrolled in Medicaid or Medicare. Study results also may not be applicable to patients treated with lisdexamfetamine, especially those with co-morbid binge-eating disorder, or to patients using the study medications off-label or for a labeled use other than ADHD.

Third, errors and omissions may occur in coding of diagnoses or procedures; however, there is no reason to believe that these disproportionately affected particular age groups. Similarly, a number of different classification symptoms could reasonably have been used to define serious CVD, and any clinical classification system based on claims data may be imperfect. However, findings of the present study were consistent with those of previous studies of CVD and related co-morbidities in adults treated with pharmacotherapy for ADHD [30, 31].

Fourth, claims data generally do not indicate severity of illness, although proxy measures (e.g., a hospital stay for CVD) and certain diagnoses (e.g., a diagnosis of congestive heart failure) do provide some indication of level of risk. Most notably, it is not possible from the present study to determine the severity of ADHD, or the benefit-versus-risk ratio of treating ADHD with pharmacotherapy for any individual patient.

Fifth, diagnoses representing addiction/abuse in the present study were not specific as to particular substance, partly because of limitations of diagnostic coding, and partly because patients with addictions to one substance may be predisposed to abuse of others (i.e., the phenomenon known as "addiction transfer") $[55,56]$. Additionally, because of uncertainty in diagnostic coding in claims data, particularly for conditions that are either stigmatized or difficult to diagnose, we reported rates of addiction/abuse in a range that reflects a sensitivity analysis around nonspecific codes for long-term medication/opiate use. Because diagnostic coding is generally more complete in inpatient than outpatient settings [57], and because $97 \%$ of inpatient claims with the nonspecific code also had a specific diagnosis code for addiction/abuse, we believe that the true rate of addiction is probably closer to the upper end than the lower end of our estimate. However, there is no "gold standard" method to identify non-medical use of abusable drugs in automated claims data [58]. Further research should investigate this issue in an attempt to produce a more precise estimate of addiction/abuse prevalence among adults using stimulants for ADHD.

\section{Conclusions}

In a commercially insured sample of US adults treated with stimulants or atomoxetine for ADHD, prevalence of preexisting serious CVD was $3.6 \%$ among those aged 45-54 years and 7.2\% among those aged 55-64 years.
Among adults treated with stimulants, $11.3-18.5 \%$ were diagnosed with abuse or addiction in the 12 months prior to the start of ADHD pharmacotherapy. Future research should assess possible harms associated with potentially contraindicated uses of ADHD pharmacotherapy by adults, particularly in those at highest baseline risk of adverse drug events.

\section{Compliance with Ethical Standards}

Funding This work was funded by Midwestern University with no external support or funding.

Conflicts of interest KAF, LED, AMP, and DAS have no financial or other conflicts of interest in the subject of this manuscript.

Open Access This article is distributed under the terms of the Creative Commons Attribution-NonCommercial 4.0 International License (http://creativecommons.org/licenses/by-nc/4.0/), which permits any noncommercial use, distribution, and reproduction in any medium, provided you give appropriate credit to the original author(s) and the source, provide a link to the Creative Commons license, and indicate if changes were made.

\section{References}

1. Olfson M, Blanco C, Wang S, Greenhill LL. Trends in officebased treatment of adults with stimulants in the United States. J Clin Psychiatry. 2013;74(1):43-50.

2. Robison LM, Sclar DA, Skaer TL. Datapoints: trends in ADHD and stimulant use among adults: 1995-2002. Psychiatr Serv. 2005;56(12):1497-8.

3. Robison LM, Skaer TL, Sclar DA. Is attention-deficit hyperactivity disorder (ADHD) diagnosed in adults? Int J Pharm Med. 2004;18(6):337-41.

4. Paris J, Bhat V, Thombs B. Is adult attention-deficit hyperactivity disorder being overdiagnosed? Can J Psychiatry. 2015;60(7):324-8.

5. Sclar DA, Robison LM, Castillo LV, et al. Attention deficit/hyperactivity disorder among adults in the United States: trends in diagnosis and use of pharmacotherapy. Pharm Med. 2012;26(2):97-101.

6. Fairman KA, Peckham AM, Sclar DA. Diagnosis and treatment of ADHD in the United States. J Atten Disord. 2017. https://doi. org/10.1177/1087054716688534.

7. American Psychiatric Association. DSM history. Changes from DSM-IV-TR to DSM-5. 2013. https://www.psychiatry.org/File\% 20Library/Psychiatrists/Practice/DSM/APA_DSM_Changes_ from_DSM-IV-TR_-to_DSM-5.pdf. Accessed 09 Oct 2017.

8. CenterWatch. FDA-approved drugs by medical condition. Attention deficit/hyperactivity disorder. 2017. http://www. centerwatch.com/drug-information/fda-approved-drugs/medicalconditions/. Accessed 09 Oct 2017.

9. Hodgkin D, Horgan CM, Quinn AE, Merrick E, Stewart MT, Leslie LK. Management of newer medications for attention-deficit hyperactivity disorder in commercial health plans. Clin Ther. 2014;36(12):2034-46.

10. Martinez-Raga J, Knecht C, Szerman N, Martinez MI. Risk of serious cardiovascular problems with medications for attentiondeficit hyperactivity disorder. CNS Drugs. 2013;27:15-30. 
11. Post RE, Kurlansik SL. Diagnosis and management of adult attention-deficit/hyperactivity disorder. Am Fam Physician. 2012;85(9):890-6.

12. Subcommittee on Attention-Deficit/Hyperactivity Disorder; Steering Committee on Quality Improvement and Management, Wolraich M, Brown L, Brown RT, et al. ADHD: clinical practice guideline for the diagnosis, evaluation, and treatment of attention-deficit/hyperactivity disorder in children and adolescents. Pediatrics. 2011;128(5):1007-22.

13. U.S. Food and Drug Administration. FDA drug safety communication: safety review update of medications used to treat attention-deficit/hyperactivity disorder (ADHD) in adults. December 12, 2011. http://www.fda.gov/Drugs/DrugSafety/ ucm279858.htm. Accessed 09 Oct 2017.

14. Adderall $\mathrm{XR}^{\circledR}$ [package insert]. Lexington, MA: Shire US Inc.; 2015. https://www.accessdata.fda.gov/drugsatfda_docs/label/ 2013/021303s0261bl.pdf. Accessed 09 Oct 2017.

15. Zenzedi ${ }^{\circledR}$ [package insert]. Atlanta, GA: Arbor Pharmaceuticals, LLC. $2017.2 \mathrm{http} / / / \mathrm{www} . z e n z e d i . c o m / d o c s /$ PIandMedicationGuide.pdf. Accessed 09 Oct 2017.

16. Ritalin ${ }^{\circledR}$ [package insert]. East Hanover, NJ: Novartis Pharmaceuticals Corporation. 2017. https://www.pharma.us.novartis.com/ sites/www.pharma.us.novartis.com/files/ritalin_ritalin-sr.pdf. Accessed 09 Oct 2017.

17. Focalin ${ }^{\circledR}$ [package insert]. East Hanover, NJ: Novartis Pharmaceuticals Corporation. 2017. https://www.pharma.us.novartis. com/sites/www.pharma.us.novartis.com/files/focalin.pdf. Accessed 09 Oct 2017.

18. Strattera ${ }^{\circledR}$ [package insert]. Indianapolis, IN: Eli Lilly and Company. 2017. http://pi.lilly.com/us/strattera-pi.pdf. Accessed 09 Oct 2017.

19. Goff DC, Lloyd DM, Bennett G, et al. 2013 ACC/AHA guideline on the assessment of cardiovascular risk: a report of the American College of Cardiology/American Heart Association Task Force on Practice Guidelines. J Am Coll Cardiol. 2014;63(25 Pt B):2935-59.

20. Sinha A, Lewis O, Kumar R, Yeruva SL, Curry BH. Adult ADHD medications and their cardiovascular implications. Case Rep Cardiol. 2016;2016:2343691. https://doi.org/10.1155/2016/ 2343691.

21. U.S. National Institute on Drug Abuse. Stimulant ADHD medications: methylphenidate and amphetamines. DrugFacts. 2014. https://www.drugabuse.gov/publications/drugfacts/stimulant-adhdmedications-methylphenidate-amphetamines. Accessed 09 Oct 2017.

22. Martinez-Raga J, Ferreros A, Knecht C, de Alvaro R, Carabal E. Attention-deficit hyperactivity disorder medication use: factors involved in prescribing, safety aspects and outcomes. Ther Adv Drug Saf. 2017;8(3):87-99.

23. Substance Abuse and Mental Health Services Administration, Drug Abuse Warning Network. Emergency department visits involving non-medical use of central nervous system stimulants among adults aged 18 to 34 increased between 2005 and 2011. 2013. https://www.samhsa.gov/data/sites/default/files/spot103cns-stimulants-adults/spot103-cns-stimulants-adults.pdf. Accessed 09 Oct 2017.

24. Substance Abuse and Mental Health Services Administration, Drug Abuse Warning Network. Emergency department visits involving attention deficit/hyperactivity disorder stimulant medications. 2013. https://www.samhsa.gov/data/sites/default/files/DAWN073/DAW N073/sr073-ADD-ADHD-medications.htm. Accessed 09 Oct 2017.

25. Barry CL, Martin A, Busch SH. ADHD medication use following FDA risk warnings. $J$ Ment Health Policy Econ. 2012;15(3):119-25.

26. Starner CI, Schafer JA, Heaton AH, Gleason PP. Rosiglitazone and pioglitazone utilization from January 2007 through May 2008 associated with five risk-warning events. J Manag Care Pharm. 2008;14(6):523-31.

27. Gleason PP, Phillips J, Fenrick BA, Delgado-Riley A, Starner CI. Dalfampridine prior authorization program: a cohort study. J Manag Care Pharm. 2013;19(1):18-25.

28. Sclar DA, Robison LM, Castillo LV, et al. Concomitant use of triptan, and SSRI or SNRI after the US Food and Drug Administration alert on serotonin syndrome. Headache. 2012;52(2):198-203.

29. Anand K, Sketris I, Zhang Y, Levy A, Gamble JM. The impact of US FDA and Health Canada warnings related to the safety of high-dose simvastatin. Drugs Real World Outcomes. 2017;4:215-23.

30. Gerhard T, Winterstein AG, Olfson M, Huang C, Saidi A, Crystal S. Pre-existing cardiovascular conditions and pharmacological treatment of adult ADHD. Pharmacoepidemiol Drug Saf. 2010;19(5):457-64.

31. Habel LA, Cooper WO, Sox CM, et al. ADHD medications and risk of serious cardiovascular events in young and middle-aged adults. JAMA. 2011;306(24):2673-83.

32. Holick CN, Turnball BR, Jones ME, et al. Atomoxetine and cerebrovascular outcomes in adults. J Clin Psychopharmacol. 2009;29:453-60.

33. Schelleman H, Bilker WB, Kimmel SE, et al. Methylphenidate and risk of serious cardiovascular events in adults. Am J Psychiatry. 2012;169:178-85.

34. MarketScan studies: abbreviated bibliography. 2017. http:// truvenhealth.com/markets/life-sciences/products/data-tools/market scan-bibliography. Accessed 09 Oct 2017.

35. Vyvanse ${ }^{\circledR}$ [package insert]. Lexington, MA: Shire US Inc.; Revised 2017 Jan. http://pi.shirecontent.com/PI/PDFs/Vyvanse_ USA_ENG.pdf. Retrieved 11 Oct 2017.

36. Garvey WT, Mechanick JI, Brett EM, Garber AJ, Hurley DL, Jastreboff AM, Nadolsky K, Pessah-Pollack R, Plodkowski R; Reviewers of the AACE/ACE Obesity Clinical Practice Guidelines. American Association of Clinical Endocrinologists and American College of Endocrinology comprehensive clinical practice guidelines for medical care of patients with obesity. Endocr Pract. 2016;22(Suppl 3):1-203.

37. Olguin P, Fuentes M, Gabler G, Guerdjikova AI, Keck PE Jr, McElroy SL. Medical comorbidity of binge eating disorder. Eat Weight Disord. 2017;22(1):13-26.

38. Centers for Medicare \& Medicaid Services. Stimulant and related medications: U.S. Food and Drug Administration-approved indications and dosages for use in adults. 2017. https://www.cms.gov/ Medicare-Medicaid-Coordination/Fraud-Prevention/MedicaidIntegrity-Education/Pharmacy-Education-Materials/Downloads/ stim-adult-dosingchart11-14.pdf. Accessed 07 Dec 2017.

39. Drucker AM, Qureshi AA, Amand C, Villeneuve S, Gadkari A, Chao J, Kuznik A, Bégo-Le-Bagousse G, Eckert L. Health care resource utilization and costs among adults with atopic dermatitis in the United States: a claims-based analysis. J Allergy Clin Immunol Pract. 2017. https://doi.org/10.1016/j.jaip.2017.10.024.

40. Bonafede M, Cai Q, Cappell K, Kim G, Sapra SJ, Shan N, et al. Factors associated with direct health care costs among patients with migraine. J Manag Care Pharm. 2017;23(11):1169-76.

41. Choong CK, Ford JH, Nyhuis AW, Joshi SG, Robinson RL, Aurora SK, Martinez JM. Clinical characteristics and treatment patterns among patients diagnosed with cluster headache in U.S. healthcare claims data. Headache. 2017;57(9):1359-74.

42. Hong KN, Fuster V, Rosenson RS, Rosendorff C, Bhatt DL. How low to go with glucose, cholesterol, and blood pressure in primary prevention of CVD. J Am Coll Cardiol. 2017;70(17):2171-85.

43. ICD9Data.com. 2015 ICD-9-CM diagnosis code V58.69. 2017. http://www.icd9data.com/2015/Volume1/V01-V91/V50-V59/V58/ V58.69.htm. Accessed 07 Dec 2017. 
44. ICD10Data.com. ICD-10-CM diagnosis code Z79.891. 2017. http://www.icd10data.com/ICD10CM/Codes/Z00-Z99/Z77-Z99/ Z79-/Z79.891. Accessed 07 Dec 2017.

45. Rost K, Smith R, Matthews DB, Guise B. The deliberate misdiagnosis of major depression in primary care. Arch Fam Med. 1994;3(4):333-7.

46. Fugelstad A, Ramstedt M, Thiblin I, Johansson LA. Drug-related deaths: statistics based on death certificates miss one-third of cases. Scand J Public Health. 2017. https://doi.org/10.1177/ 1403494817745187.

47. Kisely S, Lin E, Gilbert C, Smith M, Campbell LA, Vasiliadis HM. Use of administrative data for the surveillance of mood and anxiety disorders. Aust NZ J Psychiatry. 2009;43(12):1118-25.

48. Coding Pro. V58.69. 2017. http://www.coding-pro.com/icd-9/ code_details.php?icd_code=V5869. Accessed 07 Dec 2017.

49. IBM SPSS. Mantel-Haenszel test for trend available in crosstabs. 2016. http://www-01.ibm.com/support/docview.wss?uid= swg21477269. Accessed 09 Oct 2017.

50. Levi J, Segal LM, Miller AF. Prescription drug abuse: strategies to stop the epidemic. 2013. Trust for America's Health. October 2013. http://healthyamericans.org/assets/files/TFAH2013RxDrugAbuse Rpt16.pdf. Accessed 09 Oct 2017.

51. McCabe SE, West BT, Teter CJ, et al. Trends in medical use, diversion, and non-medical use of prescription medications among college students from 2003 to 2013: connecting the dots. Addict Behav. 2014;39(7):1176-82.
52. Fatseas M, Debrabant R, Auriacombe M. The diagnostic accuracy of attention-deficit/hyperactivity disorder in adults with substance use disorders. Curr Opin Psychiatry. 2012;25(3):219-25.

53. Kolar D, Keller A, Golfinopoulos M, Cumyn L, Syer C, Hechtman L. Treatment of adults with attention-deficit/hyperactivity disorder. Neuropsychiatr Dis Treat. 2008;4(2):389-403.

54. Nissen SE. ADHD drugs and cardiovascular risk. N Engl J Med. 2006;354(14):1445-8.

55. Blum K, Sheridan PJ, Wood RC, et al. The D2 dopamine receptor gene as a determinant of reward deficiency syndrome. J R Soc Med. 1996;89(7):396-400.

56. Bowirrat A, Oscar-Berman M. Relationship between dopaminergic neurotransmission, alcoholism, and reward deficiency syndrome. Am J Med Genet. 2005;132B(1):29-37.

57. Hicks J. The major differences between physician and hospital billing: how medical biller job duties differ between settings. Verywell.com. November 7, 2017. https://www.verywell.com/ differences-between-physician-hospital-billing-2317429. Accessed 07 Dec 2017.

58. Canan C, Polinski JM, Alexander GC, Kowal MK, Brennan TA, Shrank WH. Automable algorithms to identify non-medical opioid use using electronic data: a systematic review. JAMIA. 2017;24(6):1204-10. 